\title{
Dispersion management in nanorod metamaterials (Conference Presentation) (Withdrawal Notice)
}

Tomasz Stefaniuk, R. Margoth Córdova-Castro, Mazhar Nasir, Anatoly Zayats

Tomasz Stefaniuk, R. Margoth Córdova-Castro, Mazhar E. Nasir, Anatoly V. Zayats, "Dispersion management in nanorod metamaterials (Conference Presentation) (Withdrawal Notice)," Proc. SPIE 10671, Metamaterials XI, 106710P (2 April 2021); doi: 10.1117/12.2309513

SPIE. Event: SPIE Photonics Europe, 2018, Strasbourg, France 


\section{Dispersion management in nanorod metamaterials (Conference Presentation) (Withdrawal Notice)}

Tomasz Stefaniuk, ${ }^{1,2}$ R. Margoth Córdova-Castro, ${ }^{1}$ Mazhar E. Nasir, ${ }^{1}$ Anatoly V. Zayats ${ }^{1}$

${ }^{1}$ King's College London (United Kingdom) ${ }^{2}$ Univ. of Warsaw (Poland)

Proc. SPIE 10671, 106710P (2018)

Online Publication Date: 23 May 2018

Withdrawn from Publication: 2 April 2018

Conference Date: 22 March-26 March 2018

Conference Location: Strasbourg, France

Conference Title: Metamaterials XI

Conference Chairs: Allan D. Boardman, Anatoly V. Zayats, Kevin F. MacDonald

Publisher's Note: This video, originally published on 23 May 2018, was withdrawn per author request. 